\title{
Stability of multi-component epilayers and nanopattern formation
}

\author{
Dongchoul Kim and Wei Lu* \\ Department of Mechanical Engineering, University of Michigan, Ann Arbor, MI 48109, USA; *Author for \\ correspondence (Tel.: +1-734-647-7858; Fax: +1-734-647-3170; E-mail: weilu@umich.edu)
}

Received 30 September 2003; accepted in revised form 15 September 2004

Key words: nanostructure, linear stability analysis, concentration modulation, ternary epilayer, selfassembly, surface stress

\begin{abstract}
A uniform multi-component epilayer may lose stability under the combined action of spinodal decomposition and epilayer-substrate interaction, separating into multiple phases. The phases may further selforganize into regular patterns. This paper investigates the compositional stability of a ternary epliayer and the subsequent emergence of nanoscale patterns. Multiple energetic forces and kinetic processes involving phase separation, phase coarsening and phase refining are incorporated into a continuous phase field model. Linear stability analysis is performed by perturbing a uniform concentration field into a sinusoidal field with small amplitude and arbitrary wavelength. The analysis shows that the epilayer-substrate interaction counteracts the coarsening effect of phase boundary energy and may lead to the formation of steady nanoscale patterns. Detailed analysis also reveals the interaction of multi-phases and its effect on the stability condition. Numerical simulation of evolving concentration field is discussed at the end of the paper. The simulations show that the pattern formation process of multi-component epilayers involves remarkably rich dynamics.
\end{abstract}

\section{Introduction}

Experimental evidence has accumulated in recent decades that atoms may diffuse on solid surfaces and self-organize into ordered nanoscale patterns. For instance, Kern et al. (1991) showed that a submonolayer of oxygen on a $\mathrm{Cu}\left(\begin{array}{lll}1 & 1 & 0\end{array}\right)$ surface could form stable periodic stripes of alternating oxygen overlayer and bare copper. The stripes had a width of about $10 \mathrm{~nm}$ and run in the $<001>$ direction. Pohl et al. (1999) deposited a mixture of

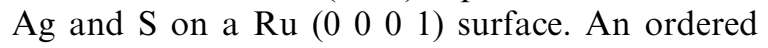
triangular lattice of $\mathrm{S}$ rich dots formed in the $\mathrm{Ag}$ matrix. The size of the dots was about $3.4 \mathrm{~nm}$. Similar ordered patterns were observed on $\mathrm{Cu}$ (111) surfaces covered with a submonolayer of Ag (Yoshimura et al., 2000) or $\mathrm{Na}$ (Kliewer \&
Berndt, 2001). Plass et al. (2001) showed that a monolayer of $\mathrm{Cu}$ and $\mathrm{Pb}$ on a $\mathrm{Cu}\left(\begin{array}{lll}1 & 1 & 1\end{array}\right)$ surface could form ordered patterns of dots or stripes, depending on the percentage of $\mathrm{Pb}$ atoms in the epilayer. Many ternary semi-conductor epilayers demonstrated lateral composition modulation, such as (In,Ga)P on GaAs (Follstaedt et al., 1994), AlInAs on InP (Francoeur et al., 2002) and GaAsN on GaAs (Suemune et al., 2000). The typical feature size is in the range of 10-50 nm.

The observations are intriguing and call for a study of the compositional stability and pattern emergence mechanism of multi-component epilayers. Spinodal decomposition and phase coarsening in a bulk material system is well known. Consider a bulk two-phase alloy. When the alloy is annealed, atoms diffuse to reduce the surface area 
and the corresponding surface energy. The phases will coarsen until only one large particle is left in the matrix. However, the situation is different for a multi-phase epilayer on an elastic substrate, where the surface stress causes a phase refining action (Alerhand et al., 1988; Ng \& Vanderbilt, 1995; Lu \& Suo, 1999; Suo \& Lu, 2000). This refining action competes with coarsening due to interfacial energy. As a result, the phases reach an equilibrium size. Moreover, the competition of two actions minimizes the total free energy, and causes the system self-assemble into superlattices.

We recently developed a phase field model for binary epilayers (Lu \& Suo, 1999, 2001, 2002a, 2002b). The epilayer is treated as a superficial object, whose concentration field evolves over time. Our approach is different from that of Guyer and Voohees (1998) and Glas (1997). They treated the case where an epilayer can be considered as a semi-infinite bulk. Their models did not have the ingredient of phase refining since the film thickness effect is not included. The work of Vanderbilt and co-workers on surface reconstruction (Alerhand et al., 1988; Ng \& Vanderbilt, 1995) highlighted the refining action of non-uniform surface stress, which was induced by the anisotropy of structure variants. For a multi-component epilayer, the surface stress nonuniformity originates from composition modulation, rather than structure variants. In addition, the free energy of mixing plays significant roles, which may be potent enough to stabilize a uniform epilayer. Unlike Vanderbilt and co-workers, we do not preassume the pattern types. Our model is a dynamic model, and the system can generate whatever patterns it favors. This capability is important to study the growth of complicated multi-component systems, which involves remarkably rich dynamics.

This paper extends our study from binary epilayers to ternary epilayers. The focus of this paper is stability, pattern emergence, and multi-phase effect. Section 'A phase field model' develops a phase field model for ternary epilayers. Section 'Linear stability analysis' specializes the model to one-dimensional composition variation, and examines the stability of a uniform ternary epilayer against small perturbations in the concentration field. Discussions about the numerical simulation and results are presented in Section 'Numerical simulation'.

\section{A phase field model}

This section develops a continuous phase field model for a ternary epilayer on a solid substrate. Consider an epilayer composed three kinds of atomic species A, B, and $\mathrm{C}$ on a substrate of atomic species S. As shown in Figure 1, the epilayer is taken to be an infinite large surface and the substrate a semi-infinite elastic body. The substrate occupies the half-space below the $x_{1}-x_{2}$ plane $\left(x_{3} \leq 0\right)$. We assume that the epilayer is a substitutional alloy of species A, B and C, and the atomic diffusion is confined within the epilayer.

The free energy of the system consists of the surface energy plus the bulk elastic energy, namely,

$$
G=\int \Gamma \mathrm{d} A+\int W \mathrm{~d} V,
$$

where $\Gamma$ is the surface energy per unit area of the epilayer, and $W$ the elastic energy per unit volume of the substrate. The area and volume are measured in the undeformed configuration of an infinite substrate. We denote the atomic fraction of component $\mathrm{A}$ and $\mathrm{B}$ in the epilayer by $C_{1}$ and $C_{2}$, respectively. The fraction of atomic sites in the epilayer occupied by species $\mathrm{C}$ is $1-C_{1}-C_{2}$ since the epilayer is a substitutional alloy. We assume the concentration fields $C_{1}\left(x_{1}, x_{2}, t\right)$ and $C_{2}\left(x_{1}, x_{2}, t\right)$ are time-dependent and spatially continuous. For an isotropic and linearly elastic substrate, $W$ is a quadratic function of strain. The material parameters are Young's modulus and Poisson's ratio. Generally speaking, the surface energy per unit area, $\Gamma$, is a function of the concentration $C_{1}, C_{2}$, the concentration gradient $C_{1, \alpha}$, $C_{2, \alpha}$, and the strain in the surface, $\varepsilon_{\alpha \beta}$ (A Greek subscript runs from 1 to 2 ). Hence, the surface energy can be expanded by

$$
\begin{aligned}
\Gamma & =g+h_{11} C_{1, \alpha} C_{1, \alpha}+h_{12} C_{1, \alpha} C_{2, \alpha} \\
& +h_{22} C_{2, \alpha} C_{2, \alpha}+f \varepsilon_{\beta \beta},
\end{aligned}
$$

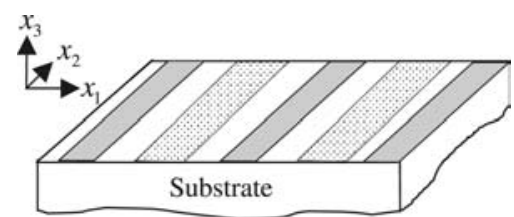

Figure 1. Geometry of the model. The epilayer is represented by an infinite surface. The substrate is represented by a semi-infinite elastic body. 
where $g, h_{11}, h_{12}, h_{22}$ and $f$ are functions of $C_{1}$ and $C_{2}$. Here we have assumed isotropy within the plane of the surface. The leading-order term in the concentration gradient is quadratic because, by symmetry, the term linear in the concentration gradient does not affect the surface energy.

When the concentration field is uniform in the epilayer, the substrate is unstrained, and the function $g\left(C_{1}, C_{2}\right)$ is the only remaining term. Thus it represents the surface energy per unit area of the uniform epilayer on the unstrained substrate. Section 'Numerical simulation' gives the expression for $g\left(C_{1}, C_{2}\right)$. The second to fourth terms in Eq. (2) represent the phase boundary energy. We assume that $h_{11}\left(C_{1}, C_{2}\right)$, $h_{12}\left(C_{1}, C_{2}\right)$ and $h_{22}\left(C_{1}, C_{2}\right)$ are constants, i.e. $h_{11}\left(C_{1}, C_{2}\right)=h_{11}, h_{12}\left(C_{1}, C_{2}\right)=h_{12}$ and $h_{22}\left(C_{1}\right.$, $\left.C_{2}\right)=h_{22}$. The phase boundary energy is always non-negative, which requires $h_{11}>0, h_{22}>0$ and $h_{12}^{2}$.

The change in the surface energy per unit strain, $f$, is known as the surface stress (Cahn, 1980; Rice \& Chuang, 1981). For simplicity, it is assumed a linear function of concentration $C_{1}$ and $C_{2}$, namely,

$$
f\left(C_{1}, C_{2}\right)=f_{0}+\phi C_{1}+\eta C_{2},
$$

where $f_{0}$ is the surface stress when the epilayer comprises pure $\mathrm{C}$ atoms. $\phi$ and $\eta$ are the slopes. When the concentration fields are non-uniform in the epilayer, the surface stress is also non-uniform, which induces an elastic field in the substrate. This elastic field drives phase refining.

The epilayer-substrate as a thermodynamic system can vary by two means: elastic displacement variation $\delta u_{i}$ and atomic relocation variation $\delta I_{\alpha}^{A}$ and $\delta I_{\alpha}^{B}$ (A Latin subscript runs from 1 to 3 ). The meaning of atomic relocation vector is briefly explained in the following. Imagine a curve in the surface of the epilayer. When some number of Aatoms cross the curve, to maintain a flat epilayer, an equal number of $\mathrm{B}$ and $\mathrm{C}$-atoms must cross in the opposite direction. Let $\boldsymbol{m}$ be the unit vector lying in the surface normal to the curve. The atomic relocation vector $\mathbf{I}^{\mathrm{A}}$ is defined such that $I_{\alpha}^{A} m_{\alpha}^{A}$ is the number of A-atoms across a unit length of the curve. The vector $\mathbf{I}^{\mathrm{B}}$ is defined in a similar manner. Due to mass conservation, the variation in the concentration fields relates to the variation in the atomic relocation by $\Lambda \delta C_{1}=-\delta I_{\alpha, \alpha}^{A}$ and $\Lambda \delta C_{2}=-\delta I_{\alpha, \alpha}^{B}$, where $\Lambda$ is the number of atoms per unit area on the surface. The energy variation of Eq. (1) gives

$$
\begin{aligned}
\delta G= & \int f \delta u_{\alpha, \alpha} \mathrm{d} A+\int \sigma_{i j} \delta u_{i, j} \mathrm{~d} V \\
& +\int \frac{\partial}{\Lambda \partial x_{\alpha}}\left(\frac{\partial g}{\partial C_{1}}+\frac{\partial f}{\partial C_{1}} \varepsilon_{\beta \beta}\right. \\
& \left.-2 h_{11} \nabla^{2} C_{1}-h_{12} \nabla^{2} C_{2}\right) \delta I_{\alpha}^{A} \mathrm{~d} A \\
& +\int \frac{\partial}{\Lambda \partial x_{\alpha}}\left(\frac{\partial g}{\partial C_{2}}+\frac{\partial f}{\partial C_{2}} \varepsilon_{\beta \beta}\right. \\
& \left.-2 h_{22} \nabla^{2} C_{2}-h_{12} \nabla^{2} C_{1}\right) \delta I_{\alpha}^{B} \mathrm{~d} A
\end{aligned}
$$

The free energy variation associated with the elastic displacement gives the classic elastic equations

$$
\sigma_{i j, j}=0
$$

in the substrate and

$$
\sigma_{3 \alpha}=\frac{\partial f}{\partial x_{\alpha}}, \quad \sigma_{33}=0
$$

on the surface. Equation (6) is the boundary condition for the elastic field in the substrate. It shows that the epilayer interacts with the substrate by boundary forces.

The free energy variation associated with the mass relocation defines the driving forces:

$$
\begin{aligned}
F_{\alpha}^{A}= & -\frac{\partial}{\Lambda \partial x_{\alpha}} \\
& \times\left(\frac{\partial g}{\partial C_{1}}+\frac{\partial f}{\partial C_{1}} \varepsilon_{\beta \beta}-2 h_{11} \nabla^{2} C_{1}-h_{12} \nabla^{2} C_{2}\right), \\
F_{\alpha}^{B}= & -\frac{\partial}{\Lambda \partial x_{\alpha}} \\
& \times\left(\frac{\partial g}{\partial C_{2}}+\frac{\partial f}{\partial C_{2}} \varepsilon_{\beta \beta}-2 h_{22} \nabla^{2} C_{2}-h_{12} \nabla^{2} C_{1}\right) .
\end{aligned}
$$

Now consider the mass conservation. In a similar way to define the mass relocation, $\mathbf{I}^{A}$, we can define a vector field $\mathbf{J}^{A}$ (called the mass flux), such that $J_{\alpha}^{A} m_{\alpha}^{A}$ is the number of $\mathrm{A}$-atoms across a unit length of the curve on the surface per unit time. The relation between $\mathbf{I}^{A}$ and $\mathbf{J}^{A}$ is analogous to that between displacement and velocity. $\mathbf{J}^{B}$ is defined in a similar way. The time rate of the concentration compensates the divergence of the flux vector, namely, 


$$
\begin{aligned}
& \Lambda \partial C_{1} / \partial t=-J_{\alpha, \alpha}^{A}, \\
& \Lambda \partial C_{2} / \partial t=-J_{\alpha, \alpha}^{B} .
\end{aligned}
$$

Finally, we specify the kinetics, namely, the rate at which the configuration changes. Following Cahn and Hilliard (1958), we assume that the atomic flux is linearly proportional to the driving force,

$$
\begin{aligned}
& J_{\alpha}^{A}=M_{1} F_{\alpha}^{A}, \\
& J_{\alpha}^{B}=M_{2} F_{\alpha}^{B} .
\end{aligned}
$$

where $M_{1}$ and $M_{2}$ are the mobilities of atoms in the epilayer.

A set of non-linear diffusion equations is obtained by combining Eqs. (5-9)

$$
\begin{aligned}
\frac{\partial C_{1}}{\partial t}= & \frac{M_{1}}{\Lambda^{2}} \nabla^{2} \\
& \times\left(\frac{\partial g}{\partial C_{1}}+\frac{\partial f}{\partial C_{1}} \varepsilon_{\beta \beta}-2 h_{11} \nabla^{2} C_{1}-h_{12} \nabla^{2} C_{2}\right), \\
\frac{\partial C_{2}}{\partial t}= & \frac{M_{2}}{\Lambda^{2}} \nabla^{2} \\
& \times\left(\frac{\partial g}{\partial C_{2}}+\frac{\partial f}{\partial C_{2}} \varepsilon_{\beta \beta}-2 h_{22} \nabla^{2} C_{2}-h_{12} \nabla^{2} C_{1}\right) .
\end{aligned}
$$

The non-uniform surface stress generates a traction field on the surface and this traction field induces an elastic field in the substrate. Cerruti (Johnson, 1985) solved the elastic field in a halfspace caused by a tangential point force acting on the surface. The elastic field generated by the distributed traction on the surface is given by a linear superposition

$$
\begin{aligned}
\varepsilon_{\beta \beta}= & -\frac{\left(1-v^{2}\right) \phi}{\pi E} \\
& \times \iint \frac{\left(x_{1}-\xi_{1}\right) \frac{\partial C_{1}}{\partial \xi_{1}}+\left(x_{2}-\xi_{2}\right) \frac{\partial C_{1}}{\partial \xi_{2}}}{\left[\left(x_{1}-\xi_{1}\right)^{2}+\left(x_{2}-\xi_{2}\right)^{2}\right]^{3 / 2}} \mathrm{~d} \xi_{1} \mathrm{~d} \xi_{2} \\
& -\frac{\left(1-v^{2}\right) \eta}{\pi E} \\
& \times \iint \frac{\left(x_{1}-\xi_{1}\right) \frac{\partial C_{2}}{\partial \xi_{1}}+\left(x_{2}-\xi_{2}\right) \frac{\partial C_{2}}{\partial \xi_{2}}}{\left[\left(x_{1}-\xi_{1}\right)^{2}+\left(x_{2}-\xi_{2}\right)^{2}\right]^{3 / 2}} \mathrm{~d} \xi_{1} \mathrm{~d} \xi_{2},
\end{aligned}
$$

where $E$ is Young's modulus and $v$ is Poisson's ratio.
Now we consider the energy of the system. The bulk elastic energy equals the work done by the shear stresses through the displacement on the surface, which gives

$$
\int W \mathrm{~d} V=\frac{1}{2} \int \sigma_{3 \beta} u_{\beta} \mathrm{d} A
$$

The non-uniform surface stress also changes the last term in Eq. (2). Thus,

$$
\begin{aligned}
\int f \varepsilon_{\beta \beta} \mathrm{d} A & =\int f u_{\beta, \beta} \mathrm{d} A \\
& =\int\left[\left(f u_{\beta), \beta}-\left(f, \beta u_{\beta}\right)\right] \mathrm{d} A .\right.
\end{aligned}
$$

The divergence theorem transforms the first term in the bracket to an integral over the boundary of the epilayer, which disappears with periodic boundary condition. The second term in the bracket, upon inserting Eq. (6), becomes

$$
\int f \varepsilon_{\beta \beta} \mathrm{d} A=-\int \sigma_{3 \beta} u_{\beta} \mathrm{d} A .
$$

Consequently, the deformation allows the nonuniform surface stress to increase bulk energy, Eq. (12), and reduce the surface energy, Eq. (14). The net result is a reduction in the total energy.

$$
\int W \mathrm{~d} V+\int f \varepsilon_{\beta \beta} \mathrm{d} A=-\frac{1}{2} \int \sigma_{3 \beta} u_{\beta} \mathrm{d} A .
$$

Therefore, a concentration-dependent surface stress by itself favors a non-uniform epilayer. The total free energy, $G$, is given by

$$
\begin{aligned}
G=\int & \left(g+h_{11} C_{1, \alpha} C_{1, \alpha}+h_{12} C_{1, \alpha} C_{2, \alpha}\right. \\
& \left.+h_{22} C_{2, \alpha} C_{2, \alpha}-\frac{1}{2} \sigma_{3 \beta} u_{\beta}\right) \mathrm{d} A
\end{aligned}
$$

\section{Linear stability analysis}

In this section, we will examine the stability of a uniform epilayer. The substrate is assumed elastically isotropic. We compare two energy states of the system. In the first sate, the epilayer has a uniform concentration field, $C_{10}$ and $C_{20}$, and the system is strain-free. Thus only the first term remains in Eq. (2). The total energy per unit area is $g\left(C_{10}, C_{20}\right)$. In the second state, the concentration 
field is perturbed from with small amplitudes $q_{1}, q_{2}$ and an arbitrary wavelength $\lambda$. Due to the isotropy, the energy of the perturbed system is independent of the direction of the wave vector. Let the direction of the wave vector coincide with the $x_{1}$ axis, as shown in Figure 1. A perturbed concentration field takes the form

$$
\left(\begin{array}{l}
C_{1}\left(x_{1}, t\right) \\
C_{2}\left(x_{1}, t\right)
\end{array}\right)=\left(\begin{array}{l}
C_{10} \\
C_{20}
\end{array}\right)+\left(\begin{array}{l}
q_{1}(t) \\
q_{2}(t)
\end{array}\right) \sin k x_{1} .
$$

The wavenumber $k$ relates to the wavelength $\lambda$ by $k=2 \pi / \lambda$. The amplitudes, $q_{1}$ and $q_{2}$, are functions of the time $t$. In the linear stability analysis, since the system is perturbed slightly from a uniform epilayer, the surface strain and the concentration gradient are small. The functions $g\left(C_{1}, C_{2}\right)$, $f\left(C_{1}, C_{2}\right), h_{11}\left(C_{1}, C_{2}\right), h_{12}\left(C_{1}, C_{2}\right)$ and $h_{22}\left(C_{1}, C_{2}\right)$ are expressed by the Taylor expansions around $C_{10}$ and $C_{20}$. As will be evident later, we only need to retain the following terms:

$$
\begin{aligned}
g\left(C_{1}, C_{2}\right)= & g\left(C_{10}, C_{20}\right)+g_{1}\left(C_{1}-C_{10}\right) \\
+ & g_{2}\left(C_{2}-C_{20}\right)+\frac{1}{2} g_{11}\left(C_{1}-C_{10}\right)^{2} \\
+ & g_{12}\left(C_{1}-C_{10}\right)\left(C_{2}-C_{20}\right) \\
+ & \frac{1}{2} g_{22}\left(C_{2}-C_{20}\right)^{2}, \\
f\left(C_{1}, C_{2}\right)= & f\left(C_{10}, C_{20}\right)+\phi\left(C_{1}-C_{10}\right) \\
& +\eta\left(C_{2}-C_{20}\right), \\
h_{11}\left(C_{1}, C_{2}\right)= & h_{11}\left(C_{10}, C_{20}\right), \\
h_{12}\left(C_{1}, C_{2}\right)= & h_{12}\left(C_{10}, C_{20}\right), \\
h_{22}\left(C_{1}, C_{2}\right)= & h_{22}\left(C_{10}, C_{20}\right) .
\end{aligned}
$$

All the expansion coefficients are material constants. Under perturbation (17), the traction boundary condition (6) becomes

$$
\sigma_{31}=\frac{\partial f}{\partial x_{1}}=\phi \frac{\partial C_{1}}{\partial x_{1}}+\eta \frac{\partial C_{2}}{\partial x_{1}}=\left(\phi q_{1}+\eta q_{2}\right) k \cos k x_{1} .
$$

The elastic field in a half-space subject to a sinusoidal surface traction can be found in Timoshenko and Goodier (1970). For our purpose, we only need the displacement on the surface, given by

$$
u_{1}=\frac{2\left(1-v^{2}\right)}{E}\left(\phi q_{1}+\eta q_{2}\right) \cos k x_{1} \text {. }
$$

The free energy can be computed from Eq. (16). Now examine the terms in turn. $g\left(C_{1}, C_{2}\right)$ is expanded into the Taylor series in Eq. (18). The contribution of $g\left(C_{1}, C_{2}\right)$ to the energy per unit area is

$$
\begin{aligned}
\frac{1}{\lambda} \int_{0}^{\lambda} g\left(C_{1}, C_{2}\right) \mathrm{d} x= & g\left(C_{10}, C_{20}\right)+\frac{1}{4} g_{11} q_{1}^{2} \\
& +\frac{1}{4} g_{12} q_{1} q_{2}+\frac{1}{4} g_{22} q_{2}^{2}
\end{aligned}
$$

The concentration gradient term in Eq. (16) contributes to the energy per unit area by

$$
\begin{gathered}
\frac{1}{\lambda} \int_{0}^{\lambda}\left[h_{11}\left(\frac{\mathrm{d} C_{1}}{\mathrm{~d} x_{1}}\right)^{2}+h_{12}\left(\frac{\mathrm{d} C_{1}}{\mathrm{~d} x_{1}}\right)\left(\frac{\mathrm{d} C_{2}}{\mathrm{~d} x_{1}}\right)\right. \\
\left.+h_{22}\left(\frac{\mathrm{d} C_{2}}{\mathrm{~d} x_{1}}\right)^{2}\right] \mathrm{d} x \\
=\frac{k^{2}}{2}\left(h_{11} q_{1}^{2}+h_{12} q_{1} q_{2}+h_{22} q_{2}^{2}\right) .
\end{gathered}
$$

Substituting Eqs. (19) and (20) into (15), we obtain the energy per unit area due to the surface stress.

$$
\frac{1}{2 \lambda} \int_{0}^{\lambda} \sigma_{31} u_{1} \mathrm{~d} x=\frac{k\left(1-v^{2}\right)}{2 E}\left(\phi q_{1}+\eta q_{2}\right)^{2} .
$$

The net free energy change due to the perturbation, $\Delta G$ (per unit area), is given by

$$
\begin{aligned}
& \Delta G=\frac{q_{1}^{2}}{2}\left\{\frac{g_{11}}{2}\left(1+\left(\frac{g_{12}}{g_{11}}\right)\left(\frac{q_{2}}{q_{1}}\right)+\left(\frac{g_{22}}{g_{11}}\right)\left(\frac{q_{2}}{q_{1}}\right)^{2}\right)\right. \\
& -\frac{\left(1-v^{2}\right) \phi^{2}}{E}\left(1+2\left(\frac{\eta}{\phi}\right)\left(\frac{q_{2}}{q_{1}}\right)+\left(\frac{\eta}{\phi}\right)^{2}\left(\frac{q_{2}}{q_{1}}\right)^{2}\right) k \\
& \left.+h_{11}\left(1+\left(\frac{h_{12}}{h_{11}}\right)\left(\frac{q_{2}}{q_{1}}\right)+\left(\frac{h_{22}}{h_{11}}\right)\left(\frac{q_{2}}{q_{1}}\right)^{2}\right) k^{2}\right\} .
\end{aligned}
$$

The stability of the system is determined by $\Delta G$. A uniform epilayer is stable against perturbations when $\Delta G$ has a positive value. We next examine the implications of Eq. (24). The surface stress term $\left(k\right.$ term) and the phase boundary term $\left(k^{2}\right.$ term) have opposite signs, suggesting a competing action. The surface stress term is more effective at long wavelengths (or small $k$ ) while the phase boundary term is more effective at short wavelengths (or large $k$ ). The competition defines a length scale 


$$
l_{1}=\frac{E h_{11}}{\left(1-v^{2}\right) \phi^{2}} .
$$

Now consider the effect of $g\left(C_{1}, C_{2}\right)$ term on stability. The surface stress term can always overcome the phase boundary term at long wavelengths. To maintain a uniform epilayer, the surface of $g\left(C_{1}, C_{2}\right)$ must be concave up, i.e. $g_{11}>0, g_{22}>0$ and $g_{12}^{2} \leq 4 g_{11} g_{22}$. When the surface is concave down, i.e. $g_{11} \leq 0, g_{22} \leq 0$ and $g_{12}^{2} 4 g_{11} g_{22}$, a concentration modulation decreases the energy. In other situations, where the surface has saddle shape, a concentration modulation may either increase or decrease the energy. The relative importance of the $g_{11}$ term is measured by a dimensionless parameter, $w$, defined as

$$
w=\frac{g_{11} l_{1}^{2}}{h_{11}}=\frac{h_{11} g_{11}}{\phi^{4}}\left(\frac{E}{1-v^{2}}\right)^{2} .
$$

Define $r=q_{2} / q_{1}$, which is the ratio of amplitudes and varies from $-\infty$ to $\infty$. In terms of $l_{1}, w$, and $r$, the free energy change, Eq. (24), can be expressed as

$$
\begin{aligned}
\Delta G=\frac{q_{1}^{2} h_{11}}{2 l_{1}^{2}} & \left\{\left(1+\left(\frac{g_{12}}{g_{11}}\right) r+\left(\frac{g_{22}}{g_{11}}\right) r^{2}\right) \frac{w}{2}\right. \\
& -\left(1+2\left(\frac{\eta}{\phi}\right) r+\left(\frac{\eta}{\phi}\right)^{2} r^{2}\right) k l_{1} \\
+ & \left.\left(1+\left(\frac{h_{12}}{h_{11}}\right) r+\left(\frac{h_{22}}{h_{11}}\right) r^{2}\right)\left(k l_{1}\right)^{2}\right\}
\end{aligned}
$$

Equation (27) involves several parameters. We consider two representative cases and focus on the effect of amplitude ratio $r$, parameter $w$ and wavelength $\lambda=2 \pi / k$ on $\Delta G$. In the first case, we take $g_{12} / g_{11}=g_{22} / g_{11}=1, h_{12} / h_{11}=h_{22} / h_{11}=1$. In the second case, we drop the cross terms and take $g_{22} / g_{11}=1, g_{12}=0, h_{22} / h_{11}=1, h_{12}=0$ and $\eta / \phi=1$.

For the first case, we can identify three situations in terms of the value of $w$.

(1) When $w>0.889, \Delta G>0$ for all wavelengths and amplitude ratio. A uniform epilayer is stable against perturbations of all wavelengths and amplitudes. Figure 2 shows the $\Delta G-\lambda$ curve for $w=0.889$. At this critical value, only the curve of $r=1$ is tangent to the line $\Delta G=0$. All

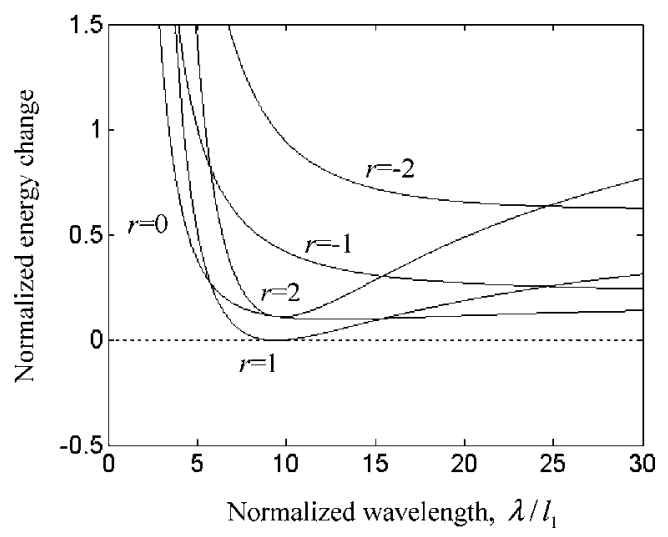

Figure 2. The free energy change, $\Delta G l_{1}^{2} / h_{11} q_{1}^{2}$, due to composition modulation is plotted as a function of the perturbation wavelength for different $r$ values. $(w=0.889)$.

curves with other amplitude ratios are above the line $\Delta G=0$ and have positive $\Delta G$.

(2) When $0<w<0.889$, the stability depends on $r$ and $\lambda$. Figure 3 shows the $\Delta G-\lambda$ curve for $w=0.5$. The curve of $r=0$ is tangent to the line $\Delta G=0$ and a curve of $r>0$ intersects with the line $\Delta G=0$ at two points. A uniform epilayer is stable against any perturbation with $r<0$. For those with $r>0$, a uniform epilayer is stable against perturbations of short and long wavelengths, but unstable against perturbations of an intermediate range of wavelengths.

(3) When $w<0$, the curve intersects with the line $\Delta G=0$ at only one point. Figure 4 shows the $\Delta G-\lambda$ curve for $w=-1$. A uniform epilayer is stable for short wavelengths but unstable for long wavelengths.

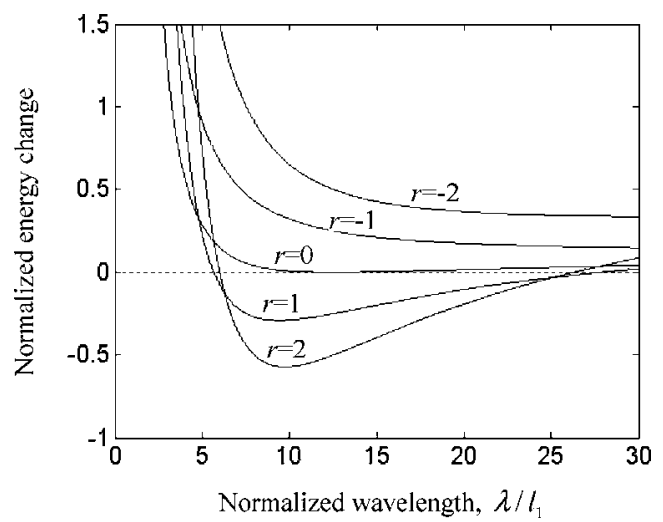

Figure 3. The free energy change, $\Delta G l_{1}^{2} / h_{11} q_{1}^{2}$, due to composition modulation is plotted as a function of the perturbation wavelength for different $r$ values. $(w=0.5)$. 


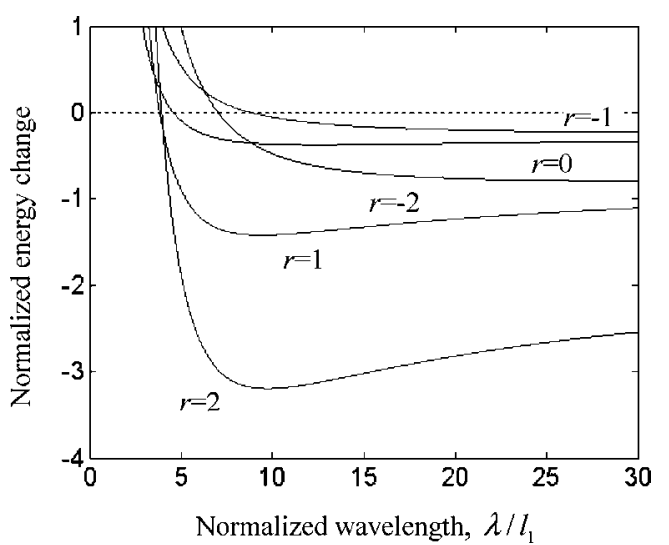

Figure 4. The free energy change, $\Delta G l_{1}^{2} / h_{11} q_{1}^{2}$, due to composition modulation is plotted as a function of the perturbation wavelength for different $r$ values. $(w=-1)$.

At long wavelength, the surface stress effect decreases the free energy when the concentration modulates, and tends to refine the epilayer. At short wavelengths, the concentration-gradient effect increases the free energy when the concentration modulates, and tends to coarsen the epilayer. Thus the free energy minimizes at some intermediate wavelength,

$$
\lambda_{m}=4 \pi l_{1}\left[\frac{1+\left(\frac{h_{12}}{h_{11}}\right) r+\left(\frac{h_{22}}{h_{11}}\right) r^{2}}{1+2\left(\frac{\eta}{\phi}\right) r+\left(\frac{\eta}{\phi}\right)^{2} r^{2}}\right] .
$$

Equation (28) is derived by taking $\partial \Delta G / \partial \lambda=0$. This wavelength provides an estimate of the feature size.

The three situations are summarized and displayed in Figure 5 on the $w-r-\lambda$ graph. The shaded surface in the graph, $w\left(r, \lambda / l_{1}\right)$, is obtained by letting $\Delta G=0$. This surface separates the space into two regions. In region above the surface, where $\Delta G>0$, the perturbed epilayer will approach to the uniform epilayer. In the other region, the uniform epilayer is unstable, and the perturbation will increase over time. To identify the region where a uniform epilayer is stable against perturbations of all amplitudes, we look at $\partial w / \partial r=0$, or

$$
\frac{\partial w}{\partial r}=\frac{2 k l_{1}\left(1-r^{2}\right)}{\left(1+r+r^{2}\right)^{2}}=0
$$

The solution of Eq. (29) is $r= \pm 1$, which is independent of the wavelength. Figure 6 shows the

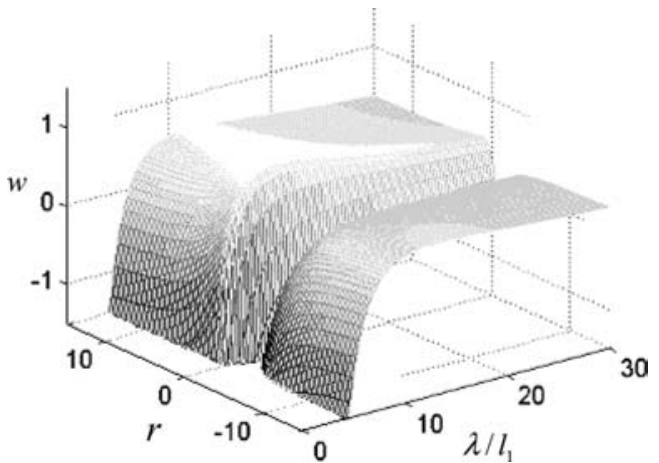

Figure 5. The $\Delta G=0$ surface in the $\mathrm{w}-\mathrm{r}-\lambda$ graph. The surface separates the space into two regions. In region above the surface, where $\Delta G>0$, the perturbed epilayer will approach to the uniform epilayer. In the other region, the uniform epilayer is unstable, and the perturbation will increase over time.

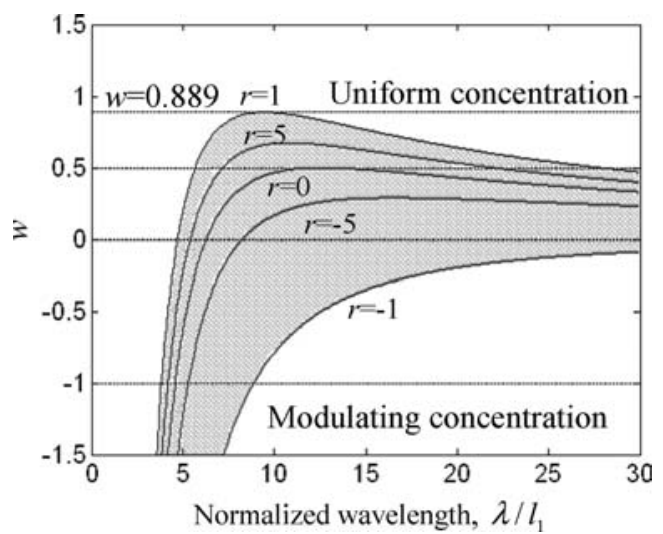

Figure 6. Diagram of stability on the $w-\lambda$ plane. Two bound curves of $r= \pm 1$ separate the plane into three regions. When $w>0.889$, a uniform epilayer is stable against all perturbations. $\left(g_{12} / g_{11}=g_{22} / g_{11}=1, \quad h_{12} / h_{11}=\right.$ $h_{22} / h_{11}=1$ and $\left.\eta / \phi=1\right)$.

$\Delta G=0$ curves for various $r$ in the $\mathrm{w}-\lambda$ plane. According to Eq. (29), the upper-bound curve is $r=1$ and the lower-bound curve is $r=-1$. All other curves stay within the shaded area bounded by the two curves, and converge to $w=0$ when the wavelength goes to infinity. The two bound curves separate the plane into three regions. A uniform epilayer is stable in the region above the $r=1$ curve, and unstable in the region below the $r=-1$ curve. In the shaded region, the stability depends on the both the wavelength and the amplitude ratio of the perturbation. In the region above $w=0.889$, a uniform epilayer is stable against all perturbations. 


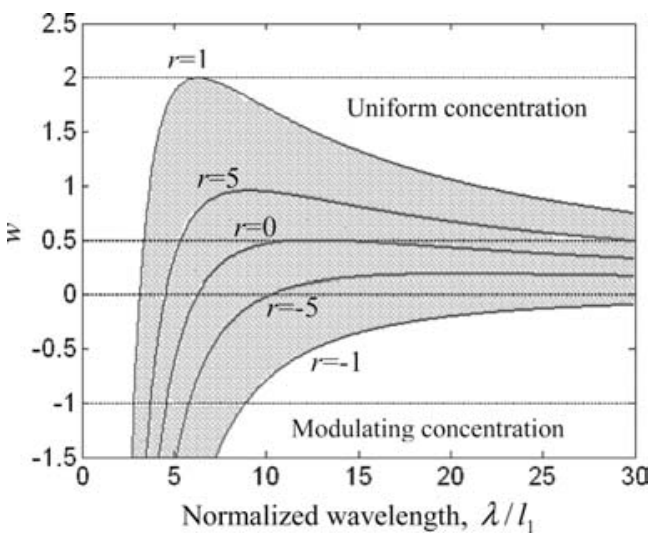

Figure 7. Diagram of stability on the $w-\lambda$ plane. Two bound curves of $r= \pm 1$ separate the plane into three regions. When $w>2$, a uniform epilayer is stable against all perturbations. $\quad\left(g_{22} / g_{11}=1, g_{12}=0, h_{22} / h_{11}=1, h_{12}=0\right.$ and $\eta / \phi=1)$.

For the second case, where $g_{22} / g_{11}=1, g_{12}=0$, $h_{22} / h_{11}=1, h_{12}=0$ and $\eta / \phi=1$, we can perform a similar analysis. Figure 7 shows the diagram of stability on the $w-\lambda$ plane. The upper-bound and lower-bound curves are still $r= \pm 1$. In the region above $w=2$, a uniform epilayer is stable against all perturbations.

The linear stability analysis is valid only when the perturbation is small and the equation can be linearized. Consequently, it cannot predict patterns after the initial stage of evolution, where the concentration non-uniformity has large magnitudes. Recently weakly non-linear stability analysis has been used to study pattern formation in the Turing patterns in the Brusselator model due to Turing and Hopf instabilities (Peña and PérezGarcía, 2001). Similar approach may be applied to study our model. However, it may not be an efficient approach to study a complexity system such as a ternary epilayer. Instead, we perform a fully non-linear numerical simulation in Fourier space as discussed below. In fact, if we only retain one Fourier component in the simulation, the problem reduces to the linear stability analysis. A weakly non-linear stability analysis is equivalent to retaining only two Fourier components in the simulation.

\section{Numerical simulation}

The pattern formation dynamics is described by a set of non-linear diffusion equations in Eq. (10). In the numerical simulation, we adopt the Muggianu's equation (Saunders \& Miodounik, 1998) for the function $g\left(C_{1}, C_{2}\right)$ :

$$
\begin{aligned}
g\left(C_{1}, C_{2}\right)= & C_{1} g_{A}+C_{2} g_{B} \\
& +\left(1-C_{1}-C_{2}\right) g_{C}+\bar{g}\left(C_{1}, C_{2}\right), \\
\bar{g}\left(C_{1}, C_{2}\right)= & \Lambda k_{\mathrm{b}} T\left\{C_{1} \ln C_{1}\right. \\
& +C_{2} \ln C_{2}+\left(1-C_{1}-C_{2}\right) \\
& \times \ln \left(1-C_{1}-C_{2}\right) \\
& +C_{1} C_{2}\left[\Omega_{12}^{0}+\Omega_{12}^{1}\left(C_{1}-C_{2}\right)\right] \\
& +C_{2}\left(1-C_{1}-C_{2}\right) \\
& \times\left[\Omega_{23}^{0}+\Omega_{23}^{1}\left(C_{1}+2 C_{2}-1\right)\right] \\
& +C_{1}\left(1-C_{1}-C_{2}\right) \\
& \left.\times\left[\Omega_{13}^{0}+\Omega_{13}^{1}\left(2 C_{1}+C_{2}-1\right)\right]\right\}
\end{aligned}
$$

where $g_{A}, g_{B}$ and $g_{C}$ are the excess energy when the epilayer is composed of pure A, B or C atoms. The average concentration is constant due to mass conservation. Hence the linear term of excess energy does not affect diffusion and can be neglected. The remaining term $\bar{g}\left(C_{1}, C_{.2}\right)$ represents the contribution from the entropy of mixing and the energy of mixing. In Eq. (30), $k_{b}$ is Boltzmann's constant and $T$ is the absolute temperature. $\Lambda$ is the number of atoms per unit area on the surface, which is mentioned before. The dimensionless numbers $\Omega_{12}^{0}, \Omega_{12}^{1}, \Omega_{23}^{0}, \Omega_{23}^{1}, \Omega_{13}^{0}$ and $\Omega_{13}^{1}$, which measure the bond strengths relative to the thermal energy $k_{\mathrm{b}} T$, control the shape of the function. Figure 8 shows the three-dimensional surface of the function $\bar{g}\left(C_{1}, C_{2}\right)$ with parameters $\Omega_{12}^{0}=2.9, \Omega_{12}^{1}=0, \Omega_{23}^{0}=2.9, \Omega_{23}^{1}=0, \Omega_{13}^{0}=2.9$ and $\Omega_{13}^{1}=0$. The surface has three wells with this parameter combination. The function $\bar{g}\left(C_{1}, C_{2}\right)$ is symmetric about $C_{1}$ and $C_{2}$ with these parameters, and reaches minimums at $\left(C_{1}, C_{2}\right)=$ $(0.116,0.116),(0.768,0.116)$, and $(0.116,0.768)$.

Before we solve the diffusion equation numerically, we normalize the length scale and the time scale. A length scale is defined by a comparison of the first and the third term in the parenthesis in Eq. (10a)

$$
b=\sqrt{\frac{h_{11}}{\Lambda k_{b} T}} .
$$




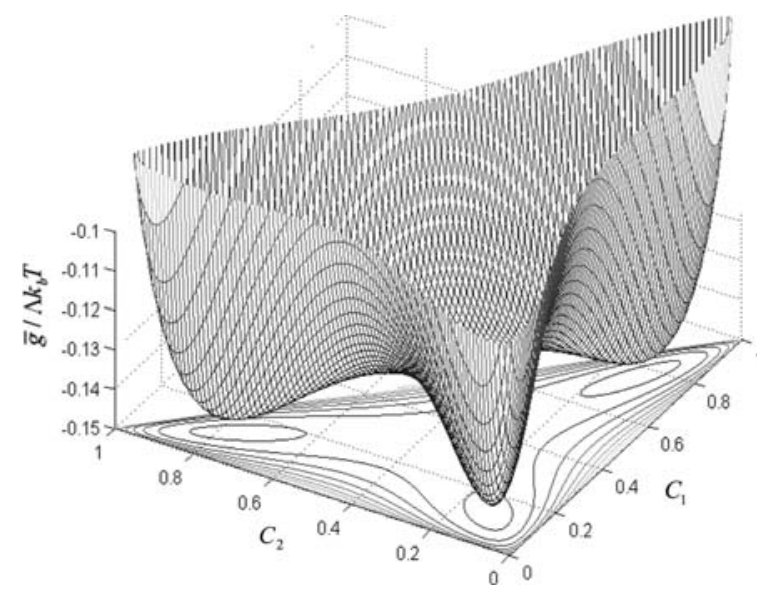

Figure 8. The three-dimensional surface of the function $\bar{g}\left(C_{1}, C_{2}\right)$ with parameters $\Omega_{12}^{0}=2.9, \Omega_{12}^{1}=0, \Omega_{23}^{0}=2.9$, $\Omega_{23}^{1}=0, \Omega_{13}^{0}=2.9$ and $\Omega_{13}^{1}=0$.

In the Cahn-Hilliard model (Cahn \& Hilliard, 1958), this length scales the distance over which the concentration changes from the level of one phase to that of the other. The magnitude of $h_{11}$ is of the order of energy per atom at a phase boundary. When we use the magnitude of $h_{11} \sim$ $1 \mathrm{eV}, \Lambda \sim 5 \times 10^{19} \mathrm{~m}^{-2}$, and $k_{b} T \sim 5 \times 10^{-21} \mathrm{~J}$ (corresponding to $T=400 \mathrm{~K}$ ), we have $b \sim 0.6 \mathrm{~nm}$. Other length scales are defined by a comparison of the second and the third term in the parenthesis in Eq. (10a and 10b):

$$
\begin{aligned}
& l_{1}=\frac{E h_{11}}{\left(1-v^{2}\right) \phi^{2}}, \quad l_{2}=\frac{E h_{11}}{\left(1-v^{2}\right) \phi \eta}, \\
& l_{3}=\frac{E h_{11}}{\left(1-v^{2}\right) \eta^{2}} .
\end{aligned}
$$

Notice that $l_{1}$ is defined in the same way as in Eq. (25). Magnitudes of $h_{11} \sim 1 \mathrm{eV}, E \sim 10^{11} \mathrm{~N} / \mathrm{m}^{2}$ which is Young's modulus of a bulk solid, and $\phi \sim 4 \mathrm{~N} / \mathrm{m}$ which is the slope of the surface stress from Ibach (1997), give $l_{1} \sim 0.6 \mathrm{~nm}$. The lengths $l_{2}$ and $l_{3}$ are of the same order. From Eq. (10a), disregarding a dimensionless factor, we note that the diffusivity scales as $D \sim M_{1} k_{\mathrm{b}} T / \Lambda$. To resolve event occurring over the length scale $b$, the time scale is $\tau=b^{2} / D$, namely,

$$
\tau=\frac{h_{11}}{M_{1}\left(k_{\mathrm{b}} T\right)^{2}} \text {. }
$$

Normalize the coordinates by $b$ and the time by $\tau$. With the dimensionless coordinates $x, \xi$ and time $t$, Eqs. $(10,11$ and 30$)$ combine into a set of normalized diffusion equations. Solving the equations by finite difference method in real space is inefficient due to the infinite integration in Eq. (11). A better method is to solve the equations by Fourier transform, which converts the integral-differential equations into regular partial differential equations. The integration operation, as well as the differentiation over space is removed and the evolution equations can be dramatically simplified. Thanks to the exponential convergence of the Fourier spectral discretization, it requires a significantly smaller number of grid points to resolve the solution to within a prescribed accuracy (Chen \& Shen, 1998).

Denote the Fourier transform of $C_{1}\left(x_{1}, x_{2}, t\right)$ by $\hat{C}_{1}\left(k_{1}, k_{2}, t\right)$, where $\left(k_{1}, k_{2}\right)$ are the coordinates in Fourier space. Then

$$
\begin{aligned}
& \hat{C}_{1}\left(k_{1}, k_{2}, t\right) \\
& =\int_{-\infty}^{\infty} \int_{\infty}^{\infty} C_{1}\left(x_{1}, x_{2}, t\right) e^{-i\left(k_{1} x_{1}+k_{2} x_{2}\right)} \mathrm{d} x_{1} \mathrm{~d} x_{2} .
\end{aligned}
$$

Taking the Fourier transform on the normalized diffusion equations gives

$$
\begin{aligned}
\frac{\partial \hat{C}_{1}}{\partial t}= & -k^{2} \hat{P}_{1}-2 k^{4} \hat{C}_{1}-k^{4} H_{1} \hat{C}_{2} \\
& +2 k^{3} Q_{1} \hat{C}_{1}+2 k^{3} Q_{2} \hat{C}_{2}, \\
\frac{\partial \hat{C}_{2}}{\partial t}= & S\left(-k^{2} \hat{P}_{2}-2 k^{4} H_{2} \hat{C}_{2}-k^{4} H_{1} \hat{C}_{1}\right. \\
& +2 k^{3} Q_{2} \hat{C}_{1}+2 k^{3} Q_{3} \hat{C}_{2},
\end{aligned}
$$

where $k=\sqrt{k_{1}^{2}+k_{2}^{2}}, \quad Q_{1}=b / l_{1}, \quad Q_{2}=b / l_{2}$, $Q_{3}=b / l_{3}, \quad S=M_{2} / M_{1}, \quad H_{1}=h_{12} / h_{11}, \quad H_{2}=$ $h_{22} / h_{11} . \hat{C}_{2}\left(k_{1}, k_{2}, t\right), \hat{P}_{1}\left(k_{1}, k_{2}, t\right)$ and $\hat{P}_{2}\left(k_{1}, k_{2}, t\right)$ are the Fourier transforms of $C_{2}\left(x_{1}, x_{2}, t\right), P_{1}\left(x_{1}, x_{2}, t\right)$ and $P_{2}\left(x_{1}, x_{2}, t\right)$. The function $P_{1}\left(C_{1}, C_{2}\right)$ comes from the derivative of $\bar{g}\left(C_{1}, C_{2}\right) / \Lambda k_{\mathrm{b}} T$ with respect to $C_{1}$ and $P_{2}\left(C_{1}, C_{2}\right)$ comes from the derivative of $\bar{g}\left(C_{1}, C_{2}\right) / \Lambda k_{\mathrm{b}} T$ with respect to $C_{2}$.

$$
\begin{aligned}
P_{1}\left(C_{1}, C_{2}\right)= & \ln \left(\frac{C_{1}}{1-C_{1}-C_{2}}\right) \\
& +C_{2}\left\{\Omega_{12}^{0}+\Omega_{12}^{1}\left(2 C_{1}-C_{2}\right)\right\} \\
& -C_{2}\left\{\Omega_{23}^{0}+\Omega_{23}^{1}\left(2 C_{1}-3 C_{2}-2\right)\right\} \\
& +\Omega_{13}^{0}\left(1-2 C_{1}-C_{2}\right)
\end{aligned}
$$




$$
\begin{aligned}
& +\Omega_{13}^{1}\left(6 C_{1}+2 C_{2}-6 C_{1}^{2}-C_{2}^{2}\right. \\
& \left.-6 C_{1} C_{2}-1\right) \\
P_{2}\left(C_{1}, C_{2}\right)= & \ln \left(\frac{C_{2}}{1-C_{1}-C_{2}}\right) \\
& +C_{1}\left\{\Omega_{12}^{0}+\Omega_{12}^{1}\left(C_{1}-2 C_{2}\right)\right\} \\
& -C_{1}\left\{\Omega_{13}^{0}+\Omega_{13}^{1}\left(3 C_{1}+2 C_{2}-2\right)\right\} \\
& +\Omega_{23}^{0}\left(1-C_{1}-2 C_{2}\right) \\
& +\Omega_{23}^{1}\left(2 C_{1}+6 C_{2}-C_{1}^{2}-6 C_{2}^{2}\right. \\
& \left.-6 C_{1} C_{2}-1\right) .
\end{aligned}
$$

The next consideration is about the time variable in Eq. (35). Instead of the explicit forward Euler method which requires very small time step to maintain stability, we employ a semi-implicit scheme proposed by Chen and Shen (1998). We treat the linear term implicitly to reduce the stability constraint, while we still treat the non-linear term explicitly to avoid solving non-linear equations at each time step. Thus, at each time step, we deal with a constant-coefficient problem.

For a given time $t$ and a time step $\Delta t$, we denote $\hat{C}_{1}^{n}=\hat{C}_{1}^{n}\left(k_{1}, k_{2}, t\right), \quad \hat{C}_{1}^{n+1}=\hat{C}_{1}\left(k_{1}, k_{2}, t+\Delta t\right), \quad P_{1}^{n}=$ $\hat{P}_{1}\left(k_{1}, k_{2}, t\right), C_{2}^{n}=\hat{C}_{2}\left(k_{1}, k_{2}, t\right), \hat{C}_{2}^{n+1}=\hat{C}_{2}\left(k_{1}, k_{2}, t+\right.$ $\Delta t)$, and $\hat{P}_{2}^{n}=\hat{P}_{2}\left(k_{1}, k_{2}, t\right)$. In Eq. (35), we replace $\hat{C}_{1} \hat{C}_{1}^{n}=\hat{C}_{2}$ by $\hat{C}_{1}^{n+1}, \partial \hat{C}_{1} / \partial t$ by $\left(\hat{C}_{1}^{n+1}-\hat{C}_{1}^{n}\right) / \Delta t$, and $\hat{P}_{1}$ by $\hat{P}_{1}^{n}$, and treat $\hat{C}_{2}, \partial \hat{C}_{2} / \partial t, \hat{P}_{2}$ in the same way. Then, we have the simulation algorithm in a discretized matrix form which is given by

$$
\begin{aligned}
\left\{\begin{array}{c}
\hat{C}_{1}^{n+1} \\
\hat{C}_{2}^{n+1}
\end{array}\right\}= & {\left[\begin{array}{cc}
1+\left(2 k^{4}-2 k^{3} Q_{1}\right) \Delta t & \left(k^{4} H_{1}-2 k^{3} Q_{2}\right) \Delta t \\
S\left(k^{4} H_{1}-2 k^{3} Q_{2}\right) \Delta t & 1+S\left(2 k^{4} H_{2}-2 k^{3} Q_{3}\right) \Delta t
\end{array}\right]^{-1} } \\
& \left.\times\left[\begin{array}{c}
\hat{C}_{1}^{n} \\
\hat{C}_{2}^{n}
\end{array}\right\}-k^{2} \Delta t\left\{\begin{array}{c}
\hat{P}_{1}^{n} \\
S \hat{P}_{2}^{n}
\end{array}\right\}\right] .
\end{aligned}
$$

For a simulation with a given time step, the coefficient matrix on the right side of Eq. (37) is constant. Hence, it is only necessary to perform the matrix inverse operation once, which is a significant benefit from the semi-implicit scheme.

The simulation is carried out in a square cell of size $L \times L$ in real space $\left(x_{1}, x_{2}\right)$. The periodic boundary condition is applied to replicate the cell to the entire surface. The cell size must be large enough to contain sufficient numbers of features but small enough to shorten the computation time. According to the linear perturbation analysis, the equilibrium wavelength is estimated as $4 \pi l_{1}$ from Eq. (28). We choose the cell size of the order $\mathrm{L} \sim 200 l_{1}$ in our simulation. The cell is divided into $N \times N$ grids. The grid space $\Delta=L / N$ should be small enough to describe the phase boundary. We choose $\Delta=b$ in our simulation. The corresponding cell size in Fourier space $\left(k_{1}, k_{2}\right)$ is $2 \pi / \Delta \times 2 \pi / \Delta$. The corresponding grid space is $2 \pi / L$. To connect the values of $C_{1}, C_{2}, P_{1}$ and $P_{2}$ at the grid points in real space to those in Fourier space, we adopt the fast Fourier transform (FFT). FFT exploits various properties of the Fourier transform to enable the transformation to be done in $O\left(N \log _{2} N\right)$ operations instead of $O\left(N^{2}\right)$ operations. The input contains the initial concentration distribution and the parameters $\mathrm{Q}_{1}, Q_{2}, Q_{3}, S, H_{1}$, $H_{2}, \Omega_{12}^{0}, \Omega_{12}^{1}, \Omega_{23}^{0}, \Omega_{23}^{1}, \Omega_{13}^{0}, \Omega_{13}^{1}$. At each time step, the values of $P_{1}^{n}, P_{2}^{n}$ at each grid point are calculated with Eq. (36). Then $C_{1}^{n}, C_{2}^{n}$ and $P_{1}^{n}, P_{2}^{n}$ are transformed to $\hat{C}_{1}^{n}, \hat{C}_{2}^{n}$ and $\hat{P}_{1}^{n}, \hat{P}_{2}^{n}$ by FFT. $\hat{C}_{1}^{n+1}$ and $\hat{C}_{2}^{n+1}$ are updated by Eq. (37). With the inverse FFT, $\hat{C}_{1}^{n+1}$ and $\hat{C}_{2}^{n+1}$ in real space are obtained. The simulation advances by repeating the procedure.

Figures 9 and 10 show selected simulation results. Each calculation cell contains $256 \times 256$ grids with grid size equal to $b$. Time is normalized by the time scale $\tau$. The time step used in the computation is $\Delta t=1.0 \times 10^{-2}$. At a given time, the concentration fields in the $\left(x_{1}, x_{2}\right)$ plane are visualized by grey scale graphs. The darker region corresponds to higher concentration and the brighter region corresponds to lower concentration. The concentration fields $C_{1}\left(x_{1}, x_{2}\right)$ and $C_{2}\left(x_{1}, x_{2}\right)$ evolve over time, but the average concentrations over the calculation cell do not change due to mass conservation. The initial conditions are given by fluctuating randomly within 0.001 from the average concentrations.

Figure 9 shows an evolution sequence from $t=0$ to 1000 . The parameters $Q_{1}, Q_{2}, Q_{3}, H_{2}$ and $S$ are taken to be $1 . H_{1}$ is taken to be 0 . Other parameters are $\Omega_{12}^{0}=2.9, \quad \Omega_{12}^{1}=0, \quad \Omega_{23}^{0}=2.9$, $\Omega_{23}^{1}=0, \Omega_{13}^{0}=2.9$ and $\Omega_{13}^{1}=0$. The left column plots the concentration field $C_{1}$ and the right column plots the concentration field $C_{2}$. The simulation starts from a random initial condition with average concentration of $C_{1}=0.4$ and $C_{2}=0.35$.

Shortly after the simulation starts, the amplitudes of the concentration fields rapidly evolve 

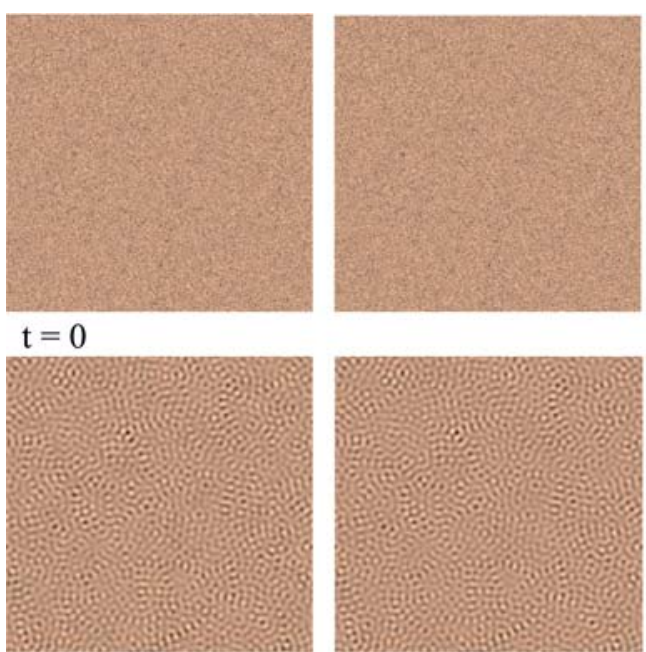

$\mathrm{t}=30$
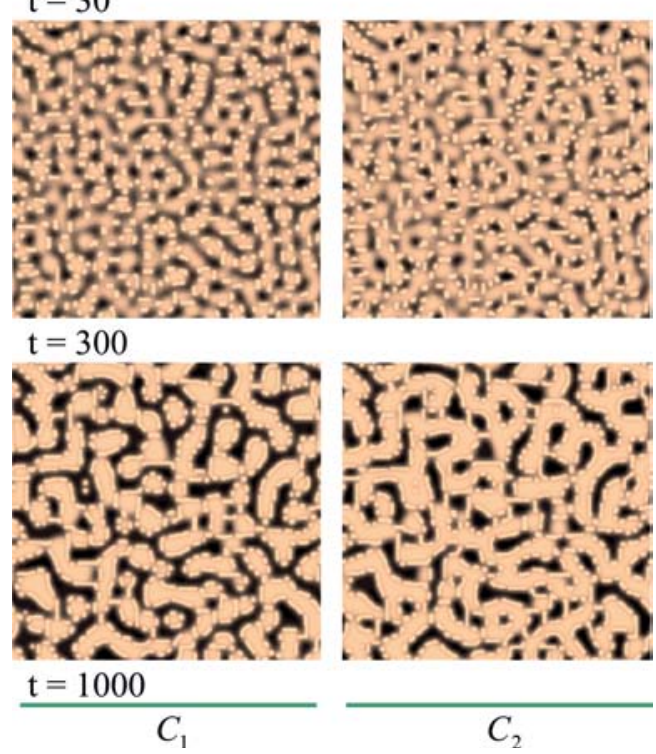

Figure 9. An evolution sequence from a random initial condition. The average concentrations of $C_{1}, C_{2}$ are 0.4 and 0.35 , respectively. $Q_{1}, Q_{2}, Q_{3}, H_{2}$ and $S$ are taken to be $1 . H_{1}$ is taken to be 0 .

close to the equilibrium values of the three phases, i.e. $(0.116,0.116),(0.768,0.116),(0.116,0.768)$. These equilibrium values are estimated by the $\bar{g}\left(C_{1}, C_{2}\right)$ term only. The phase sizes and their spatial ordering are mainly determined by the competition of phase boundary energy and elastic energy, which has only minor influence on the equilibrium compositions. At $t=300$, we can clearly identify three phases by their shades. The dark and gray phases organize into a serpentine
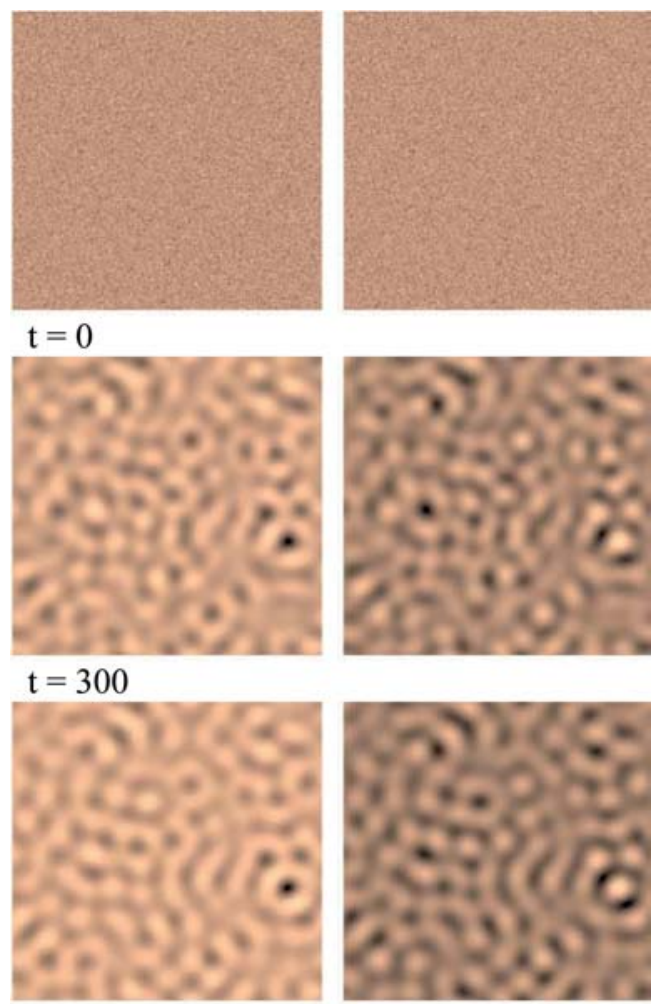

$\mathrm{t}=1000$
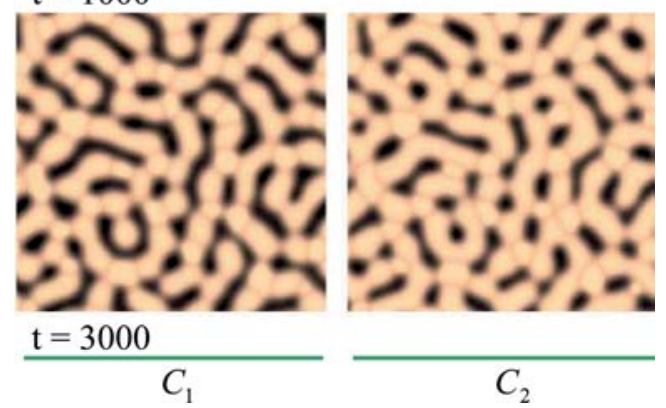

Figure 10. An evolution sequence from a random initial condition. The parameters are the same as those in Figure 9 except that $Q_{1}, Q_{2}$, and $Q_{3}$ are taken to be 0 .

structure with a bright dot phase scattering in between. In the $C_{1}$ graph, the dark, gray and bright phases have compositions close to $\left(C_{1}, C_{2}\right)=(0.768$, $0.116),(0.116,0.768)$ and $(0.116,0.116)$, respectively. The dark, gray and bright phases in the $C_{2}$ graph have compositions $(0.116,0.768),(0.768$, $0.116)$, and $(0.116,0.116)$. Compared with other two phases, the bright phase has quite small phase size (or stripe width). This can be explained in terms of the constraint of average concentration. 
Consider the concentration component $C_{3}$ of the three phases, namely $1-C_{1}-C_{2}$. The dark, gray and bright phases have $\mathrm{C}_{3}=0.116,0.116$, and 0.768 , respectively. The average concentration $(0.4$, 0.35 ), or $C_{3}=0.25$, is closer to the composition of the dark and gray phases. As a result of mass conservation, the size of the bright phase has to be smaller. At time $t=1000$, the bright dots aggregate at the boundary of the dark and gray phases, forming narrow stripes separating the two phases. The serpentine structures have been observed experimentally in many bulk or film systems.

Figure 10 shows an evolution sequences without surfaces stress, i.e. $Q_{1}=0, Q_{2}=0, Q_{3}=0$. The simulations start from the same initial conditions and average concentrations as those in Figure 9. At $t=1000$, the phases are fuzzy and hard to distinguish. The compositions are still close to the average concentration, which suggests the phase separation is far from completion at this time. However, the equilibrium compositions are already achieved at $t=300$ in Figure 9. The comparison suggests that the existence of surface stress accelerates the evolution. Without surface stress, the phases try to increase their sizes as much as possible, being restricted only by mass conservation and the size of calculation cell. The phases in Figure 10 continue to coarsen after $t=3000$, while late state evolution in Figure 9 is characterized by local reorientation. Figure 11 shows another evolution sequence. All the parameters are the same as those in Figure 9 except that $H_{1}$ is taken to be 1 . It is observed that the evolution in Figure 11 is slower compared to that in Figure 9. The simulations have demonstrated rich dynamics in the selfassembly of ternary epilayers. Additional work and simulations will be carried out to study the pattern dynamics.

\section{Concluding remarks}

This paper investigates the compositional stability of a ternary epliayer and the subsequent emergence of nanoscale patterns. We perform the linear stability analysis by perturbing a uniform concentration field into a sinusoidal field with small amplitude and arbitrary wavelength. The stability is determined by comparing the energy of a perturbed state to that of the uniform state. The analysis shows that when the free energy of mixing
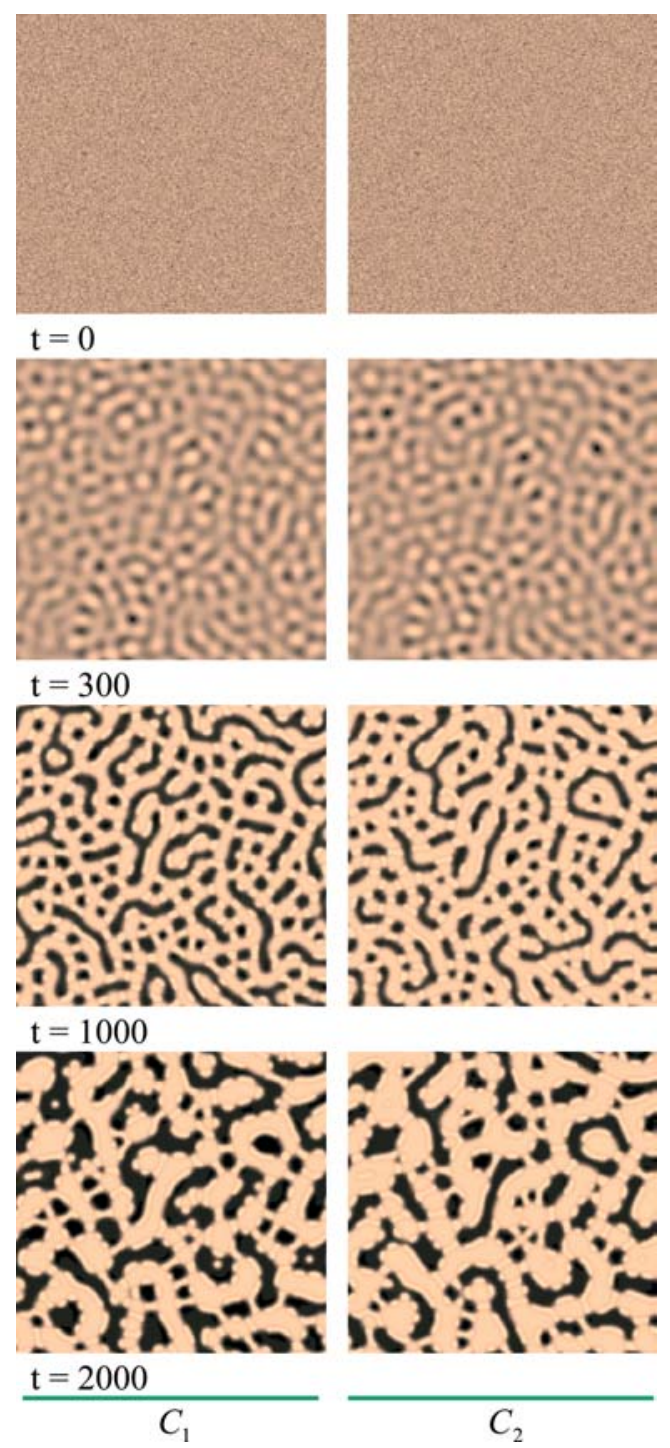

Figure 11. An evolution sequence from a random initial condition. The average concentrations of $C_{1}$ and $C_{2}$ are 0.4 and 0.35 , respectively. $Q_{1}, Q_{2}, Q_{3}, H_{1}, H_{2}$ and $S$ are taken to be 1 .

is potent enough, a uniform epilayer can be stable against any perturbation. Detailed analysis also reveals the multi-phase effect on the stability condition. Numerical simulation of evolving concentration fields is discussed at the end of this paper. Some preliminary results are presented, which suggests rich dynamics during the pattern formation of multi-component epilayers. Additional work will be reported later. 


\section{Acknowledgements}

The authors acknowledge financial support from National Science Foundation Career Award No. DMI-0348375.

\section{References}

Alerhand O.L., D. Vanderbilt, R.D. Meade \& J.D. Joannopoulos, 1988. Phys. Rev. Lett. 61, 1973.

Cahn J.W., 1980. Acta Metall. 28, 1333.

Cahn J.W. \& J.E. Hilliard 1958 J. Chem. Phys. 28, 258.

Chen L.-Q. \& J. Shen, 1998. Comp. Phys. Commun. 108, 147.

Clark P.G. \& C.M. Friend, 1999. J. Chem. Phys. 111, 6991. Follstaedt D.M., R.P. Schneider, \& E.D. Jones, 1994 J.Appl. Phys. 77, 3077.

Francoeur S., M.C.Hanna, A.G. Norman \& A. Mascarenhas, 2002. Appl. Phys. Letts. 80, 243.

Glas F., 1997. Phys. Rev. B55, 11277.

Guyer J.E. \& P.W. Voorhees, 1998. J. Crystal Growth 187, 150

Ibach H., 1997. Surf. Sci. Rep. 29, 193.
Johnson K.L., 1985. Contact Mechanics. Cambridge University Press, UK.

Kern K., H. Niebus, A. Schatz, P. Zeppenfeld, J. George \& G. Comsa, 1991. Phys. Rev. Lett. 67, 855.

Kliewer J. \& R. Berndt, 2001. Surf. Sci. 477, 250.

Lu W. \& Z. Suo, 1999. Z. Metallkunde 90, 956.

Lu W. \& Z. Suo, 2001. J. Mech. Phys. Solids 49, 1937.

Lu W. \& Z. Suo, 2002a. Phys. Rev. B65, 085401.

Lu W. \& Z. Suo, 2002b. Phys. Rev. B65, 205418.

Ng K. -O. \& D. Vanderbilt, 1995. Phys. Rev. B52, 2177.

Peña B. \& C. Pérez-García, 2001. Phys. Rev. E64, 056213.

Plass R., J.A. Last, N.C. Bartelt \& G.L. Kellogg, 2001. Nature 412, 875.

Pohl K., M.C. Bartelt, J. de la Figuera, N.C. Bartelt, J. Hrbek \& R.Q. Hwang, 1999. Nature 397, 238.

Rice J.R. \& T.-J. Chuang, 1981. J. Am. Ceram. Soc. 64, 46. Saunders N. \& N.P. Miodounik, 1998. CALPHAD-Calculation of Phase Diagrams. Pergamon New York.

Suemune I., N. Morooka, K. Uesugi, Y.-W. Ok \& T.-Y. Seong, 2000. J. Cryst. Growth 221, 546.

Suo Z. \& W. Lu, 2000. J. Mech. Phys. Solids 48, 211.

Timoshenko S.P. \& J.N. Goodier, 1970. Theory of Elasticity.McGrawHill, New York.

Yoshimura M., K. Ojima \& K. Ueda, 2000. Phys. Rev. B. 63, 35402. 